Ann. Génét. Sél. anim. I977, 9 (I), 449-45I.

Note

\title{
Les « noisillures » en race bovine Limousine
}

\author{
J. J. LAUVERGNE, \\ Département de Génétique animale, \\ Centre national de Recherches zootechniques, I.N.R.A., \\ 78350 Jouy-en-Josas
}

\begin{abstract}
Résumé
Dans le troupeau Limousin du domaine de Pompadour Corrèze, France, 7 p. cent des femelles reproductrices présentaient sur le museau de petites taches noires appelées noisillures. On pense que ces noisillures sont dues à des variations de l'expressivité de l'allèle en $A$ gouti qui donne la couleur froment à extrémités claires de la race. Ces variations pourraient être partiellement contrôlées par une hérédité de type polygénique.
\end{abstract}

La race bovine Limousine a un standard coloré qui prohibe toute tache ou pigmentation des muqueuses, qui doivent être roses (ANONYME). En fait un certain nombre d'animaux présentent des taches noires appelées " noisillures " au niveau du mufle. Dans la présente note ce phénomène est étudié à partir de données recueillies dans le troupeau du Domaine de Pompadour (Corrèze).

Chez le veau la pigmentation du mufle se présente sous forme d'un voile qui disparaît par la suite ou qui se résoud en " noisillures " qui alors généralement persistent toute la vie. La pigmentation du mufle s'accompagne parfois de pigmentation de la langue et on a noté dans un cas au moins des poils noirs autour du museau sur les oreilles et sur la tête.

Sur I03 femelles d'élevage présentes à Pompadour au printemps I977, 7 d'entre elles avaient des noisillures $(6,7 \%)$. Cinq de ces femelles accouplées à des taureaux indemnes de noisillures avaient donné 5 veaux des deux sexes présentant des noisillures et II indemnes.

Même si la ségrégation observée fait penser à une ségrégation mendélienne: $\left(\chi^{2}=2,2, \mathrm{n} . \mathrm{s}\right.$.) il est peut être hâtif de donner uneinterprétation en termes de gènes majeurs : un allèle donnant la noisillure, l'autre non. Le phénomène est sans doute plus complexe et il faudrait connaître, en premier lieu, la formule colorée exacte de la race Limousine pour laquelle on est réduit à des suppositions qui peuvent être, en gros, les suivantes. 
Chez les Bovins, à l'instar de ce qui se passe chez les autres Mammifères (SEARLE, I968), la répartition des neélanines noires et rouge dans le pelage est sous le contrôle d'allèles situés à deux loci : $A$ (Agouti) et $E$ (Extension). Le locus $E$ des Bovins semble présenter seulement deux allèles (LAUVERGNE, I977) : $E^{+}$permettant l'expressivité normale des facteurs en $A$ et $E^{d}$, noir dominant, épistatique sur $A$ et donnant un pelage noir quels que soient les allèles à ce locus. Chez le Limousin, qui est fauve à muqueuses claires, manifestement c'est $E^{+}$qui existe. Quant à l'allèle en $A$ gouti il faut dire tout d'abord que la liste des allèles à ce locus chez les bovins est loin d'être définitivement arrêtée. Elle est encore hypothétique dans plusieurs cas, mais sans doute assez longue, à l'instar de ce qui se passe dans les autres espèces de ferme ou de laboratoire (LAuvergne, I968, Misuraca et al., I973, LAUVERGNE, I977). Les allèles identifiés avec quelque certitude et qui donnent une coloration proche de celle du Limousin sont $A^{r}$ : "rouge foncé " uniforme comme en Salers ou en Hereford et $\mathrm{A}^{y}$ : "fauve à extrémité noire " comme dans l'Aubrac. Du premier, le Limousin a les muqueuses claires mais la robe est d'un rouge plus atténué et du second il a les éclaircissements sous le ventre et à l'intérieur des cuisses mais, si le froment s'approche beaucoup du fauve les muqueuses, par contre, sont claires. Il se pourrait alors :

- soit que le patron du Limousin provienne d'un autre allèle en Agouti, allèle que l'on pourrait appeler "fauve à muqueuses claires ",

-- soit qu'il ait été obtenu par une modification du phénotype donné par $A^{r}$, ou $\mathrm{A}^{y}$, ce qui est tout à fait possible car les mutants en $A$ gouti ont parfois des expressivités variables que l'on peut fixer, dans une certaine mesure, par sélection.

Dans les deux hypothèses les noisillures peuvent s'expliquer par la variabilité de l'expressivité du mutant, variabilité partiellement contrôlée par des polygènes (d'où l'allure héréditaire du phéncmène) sans qu'il soit besoin d'invoquer un gène spécialement pour la présence de taches noires sur le museau qui vont d'ailleurs quelquefois avec des poils noirs sur la tête et la pigmentation de la langue.

On peut penser que les noisillures ne risquent guère de se répandre et de se développer dans la race, même si $7 \mathrm{p}$. cent des femelles les présentent car, de toutes façons, une sélection stricte s'effectue chez les mâles.

\section{Remerciements}

Les données ont été recueillies par M. Jacques CourTors du Herd-Book Limou$\sin$ et M. Alain MaLAfosse (Union nationale des Livres généalogiques, Paris) avec la collaboration de M. AdAM du Domaine de Pompadour. Le texte a été lu par le Dr A. G. SeArle MRC Radiobiological Unit, Harwell, Didcot, Angleterre.

Reçu pour publication en décembre 1977.

\section{Summary}

The " noisillures" in Limousin cattle

Seven percent of Limousin breeding females in the Pompadour Estate, Corrèze, France exhibit small black spots on the muzzle which are refered to as "noisillures ". It is thought that these "noisillures" are a result of variations in the expressivity of the allele in the $A$ gouti series responsible for the wheat colour with light extremities observed in the breed. These variations could be partially due to a polygenic type of inheritance. 


\section{Références bibliographiques}

Anonyme. Règlement intérieur adopté par l'Assemblée Générale ordinaire du r4 décembre I973 (du Herd Book Limousin), sans date et sans référence, pp. ooo.

I.AUVERGne J. J., I966. Génétique de la couleur du pelage des Bovins domestiques (Bos taurus, Linné). Bibliogr. Genet., 20, I-68.

LaUvergne J. J., 1977. New alleles at the Agouti locus (A) in domestic Ruminants. Xth Intern. Pigment ell Conf. Abstr. c 69.

Misuraca Giovanna, Prota G., Lauvergne J. J., i974. Pigments mélaniques du pelage de quelques races bovines : le schéma possible de certaines actions géniques. Ann. Génét. Sél. anim., 6, 399-404.

SEARLE A. G., r968. Comparative Genetics of coat colour in Mammals. Logos Press, London. 